\title{
Desenvolvimento de um sistema para demonstração da projeção de Monge utilizando realidade aumentada
}

\author{
Alexandre Hering Coelho ${ }^{1}$
}

\begin{abstract}
Resumo: Nem sempre é tarefa fácil a compreensão do método de Monge no estudo da geometria descritiva. $\mathrm{O}$ entendimento dos princípios requer o poder de visualização das configurações geométricas no espaço e da transformação que ocorre para a projeção no plano. Neste trabalho é explorada a realidade aumentada para auxiliar na demonstração da projeção de Monge, de forma que as cenas geradas sejam semelhantes às encontradas em livros didáticos. Seguindo este objetivo, foi desenvolvido neste trabalho um sistema de realidade aumentada. É descrito o processo de desenvolvimento do sistema e é demonstrada a sua utilização. Como resultado foram geradas cenas onde estão representadas situações de intersecção e paralelismo, envolvendo os objetos ponto, reta e plano, simultaneamente em três e em duas dimensões. São dadas algumas considerações quanto à usabilidade do sistema.
\end{abstract}

Palavras-chave: Geometria descritiva. Projeção de Monge. Realidade aumentada.

\begin{abstract}
Not always the understanding of the Monge's method in the study of descriptive geometry is an easy task. The comprehension of the principles requires the power of visualization of geometric configurations in the space and the transformation that occurs for the projection on the plan. In this work augmented reality is explored to assist on the demonstration of the Monge's projection, in a way that the generated scenes are similar to the found on didactic books. Following this objective, an augmented reality system was developed in this work. The process of system development is described and its use is demonstrated. As result, scenes were generated where situations of intersection and parallelism are represented, involving the objects point, line and plan, simultaneously in three and in two dimensions. Some considerations about the usability of the system are given.
\end{abstract}

Keywords: Augmented reality. Descriptive geometry. Monge's projection

\section{Introdução}

Representar objetos tridimensionais em duas dimensões é de grande utilidade na engenharia. Por meio de desenhos no plano, que representam os objetos tridimensionais do mundo real, é possível efetuar a sua descrição exata. Além disso, é possível efetuar uma série de operações geométricas sobre o objeto, indiretamente em sua representação no plano. A geometria descritiva é a ciência que estuda essa representação.

O método de representação de Monge é muito explorado na geometria descritiva [1], [2], [3], [4] e [5]. Nesse método são utilizadas projeções ortogonais de objetos em dois planos perpendiculares, o plano horizontal e o plano vertical, sendo feito o rebatimento desses planos para o espaço bidimensional.

A compreensão da projeção de Monge requer a capacidade de imaginação de situações geométricas no espaço e da transformação que ocorre para a projeção no plano. De acordo com o que coloca Hugo de Andrade de Souza Júnior [2], isso não é tarefa fácil para muitos estudantes. Por isso, é interessante que sejam exploradas maneiras de facilitar essa compreensão, com o uso de recursos tecnológicos atuais que auxiliem na visualização das situações geométricas.

É possível atualmente encontrar relatos sobre o uso da realidade aumentada no ensino da geometria descritiva, tanto internacionalmente como no Brasil (seção 2.4). Porém, não foi encontrado um sistema de realidade aumentada que represente os elementos envolvidos na projeção de Monge da forma como é mostrada em livros didáticos sobre

\footnotetext{
${ }^{1}$ Florianópolis, SC, Brasil

\{ahcoelho@gmail.com\}

http://dx.doi.org/10.5335/rbca.2012.2079
}

Revista Brasileira de Computação Aplicada (ISSN 2176-6649), Passo Fundo, v. 4, n. 2, p. 2-15, out. 2012 
geometria descritiva, como, por exemplo, os descritos em Pinheiro [1], Souza Júnior [2] e Príncipe Júnior [4]. Essa forma é composta pelo desenho em perspectiva dos planos que formam a épura no espaço, sendo mostradas as linhas de projeção nesses planos dos pontos que formam os objetos, isso sempre acompanhado do desenho da épura rebatida em duas dimensões, representando a mesma situação.

Ao utilizar o livro didático para o aprendizado, é interessante que o aluno disponha de um software que lhe mostre as cenas, com diferentes configurações geométricas, tais como as que vê nos livros, podendo associar facilmente os conceitos à ideia visualizada. As cenas seriam, dessa forma, geradas a partir da interação de objetos no espaço com os planos de projeção da épura. O posicionamento e a orientação relativas entre eles seriam controlados pelo aluno movendo os objetos virtuais no espaço, como se existissem no mundo real.

O objetivo deste trabalho é a elaboração de um sistema para demonstração da projeção de Monge utilizando a técnica da realidade aumentada. As primitivas geométricas ponto, reta e plano devem ser projetadas em uma épura com o uso do sistema. As representações geradas com o sistema devem ser semelhantes às encontradas nos livros didáticos de Príncipe Júnior [4] e Souza Júnior [2], onde para cada caso analisado é mostrada a épura no espaço tridimensional (no caso, na cena de realidade aumentada) e rebatida em duas dimensões (em uma janela à parte). Deve ser explorada a geração de cenas de realidade aumentada contendo a representação de algumas propriedades básicas das projeções dos objetos.

\section{Breve revisão conceitual}

Para contextualizar o trabalho são apresentados aqui, brevemente, os conceitos de projeção de Monge e realidade aumentada. Também é colocada uma breve revisão de literatura científica sobre a utilização da realidade aumentada na geometria descritiva.

\subsection{Geometria descritiva}

Segundo Souza Júnior [2], a geometria descritiva tem por objetivo representar num plano as figuras do espaço. Ricca [5] recorre a vários métodos de representação para resolver no plano os problemas geométricos do espaço, de forma que a partir da representação possa ser gerada a figura no espaço e vice-versa.

Souza Júnior [2] coloca que tal ramo da geometria se deve ao matemático Gaspard Monge (1746-1818), militar francês que no início do século XVIII planejou um método gráfico para representação espacial que revolucionou o estudo da geometria. Príncipe Júnior [4], adicionalmente, coloca que no plano assim gerado se podem resolver todos os problemas relativos a essas figuras.

\subsection{Projeção de Monge}

A representação de Monge, ou mongeana, é um método de dupla projeção ortogonal em dois planos perpendiculares, um horizontal, denominado $\pi$, e um vertical, denominado $\pi^{\prime}$, como trazido, por exemplo, em Pinheiro [1], Príncipe Júnior [4] e Ricca [5]. A linha de intersecção desses planos é denominada linha de terra. Existem convenções para a nomenclatura adotada para pontos projetados nestes planos e para a numeração dos quatro diedros formados por eles, algumas das quais são apresentadas oportunamente no decorrer deste trabalho.

O rebatimento desses planos pela linha de terra, de forma que se tornem coincidentes, constitui o conceito de épura. Segundo Príncipe Júnior [4], épura é a representação de uma figura no espaço pelas suas projeções, estando o plano vertical rebatido sobre o horizontal. Esse conceito é sempre apresentado nos livros didáticos com o auxílio de uma figura que mostra esquematicamente o rebatimento dos planos. Esta é mostrada neste trabalho na Figura 1. Na Figura 1(a) é mostrada a representação da épura em três dimensões. O plano $\pi$ se encontra desmembrado nos planos $\pi a$ (anterior) e $\pi p$ (posterior) e o plano $\pi^{\prime}$ nos planos $\pi^{\prime} s$ (superior) e $\pi^{\prime} i$ (inferior). Os diedros são numerados em algarismos romanos de I a IV. Setas mostram o movimento de rebatimento do plano vertical sobre o horizontal. Na Figura 1(b) é mostrada a épura rebatida em duas dimensões, de forma que os planos $\pi^{\prime} s$ e $\pi p$ se tornam coincidentes, assim como os planos $\pi^{\prime} i$ e $\pi a$.

Os objetos básicos representados na épura, trazidos normalmente nos livros didáticos, são o ponto, a reta e o plano, como, por exemplo, em príncipe Júnior [4] e Souza Júnior [2]. A distância de projeção de pontos no espaço ao plano horizontal é chamada de "cota"e a "distância"ao plano vertical de "afastamento". É denominada "linha de 


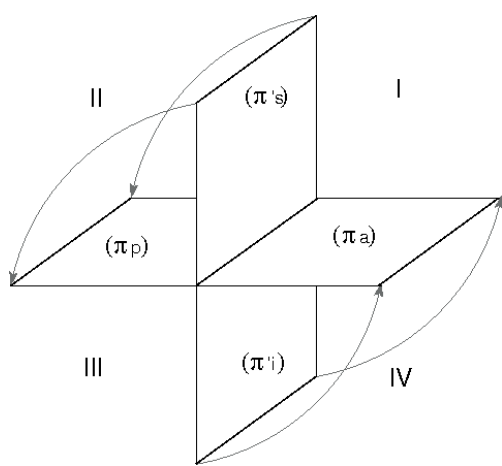

(a) Épura tridimensional, seus planos formadores e diedros

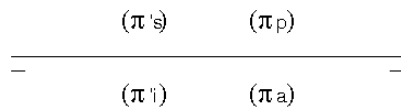

(b) Épura rebatida para duas dimensões

Figura 1: Representação do conceito de épura

projeção", ou "linha de chamada", toda linha perpendicular à linha de terra, na épura rebatida, que une as projeções de um mesmo ponto.

Com o uso da representação de Monge é possível desde a simples representação de um ponto no espaço até a realização de verificações geométricas de propriedades relativas entre as primitivas geométricas, como pertinência de ponto a reta, de reta a plano, de perpendicularismo e paralelismo entre retas e planos, verificação de verdadeira grandeza, mudanças de planos, rotação e rebatimento, relações das posições dos objetos em relação aos planos da épura, distâncias e ângulos entre objetos, representação de poliedros, representação de poliedros, secções planas e intersecções em poliedros, entre outros [4], [3], [2] e [1].

\subsection{Realidade aumentada}

De acordo com Cláudio Kirner e Tereza G. Kirner [7], realidade aumentada é a inserção de objetos virtuais no ambiente físico, mostrada ao usuário, em tempo real, com o apoio de algum dispositivo tecnológico, usando a interface do ambiente real, adaptada para visualizar e manipular os objetos reais e virtuais.

Ainda em concordância, o conceito pode ser dado como em [9]: "A realidade aumentada é a combinação de cenas do mundo real com cenas de um respectivo mundo virtual. A posição e a orientação da câmara, ou do olho humano, são medidas e transmitidas para uma câmara virtual no ambiente virtual. Esta combinação ocorre em tempo real. As cenas geradas desta forma podem dar a impressão de que os objetos contidos no mundo virtual existem no mundo real".

De acordo com Azuma [6] e Azuma, et al. [8], um sistema de visualização pode ser considerado como um sistema de realidade aumentada quando atende aos seguintes requisitos:

- combinação de mundo real com mundo virtual;

- integração de real e virtual e composição da cena em tempo real;

- referenciamento em 3D.

Como colocado por Alexandre Coelho [9], a combinação do mundo real com o mundo virtual se baseia na existência simultânea de objetos reais e virtuais em uma cena. A integração e composição da cena em tempo real significa que deve haver uma interação entre os objetos reais e virtuais, de forma que seja causada a impressão de que ambos estão sujeitos aos fenômenos físicos do mundo real. O referenciamento em 3D implica o correto posicionamento e a correta orientação dos objetos virtuais dentro do mundo real.

Segundo Coelho e Baehr [10], a idéia de visualizar objetos virtuais diretamente no mundo real foi documentada pelo norte-americano Ivan Sutherland [11] já no final dos anos 60. Esse autor demonstrou o assim chamado 
"head-mouted three dimensional display"(HMD), um equipamento acoplável à cabeça do usuário que, através de prismas e espelhos semiprateados, permitiu a visualização de objetos virtuais primitivos (formados apenas por linhas) diretamente no mundo real.

Existem diferentes maneiras de montar um sistema de realidade aumentada (SRA). A escolha parte basicamente do ambiente e do tamanho do objeto a ser analisado. Diferentes sistemas já foram elaborados seguindo as características da realidade aumentada, tendo, dentre esses, sido buscadas diferentes maneiras de supri-las, adaptando-se às finalidades específicas.

Em qualquer SRA é buscada uma taxa de atualização da cena com uma frequência suficiente para dar a impressão de ocorrer em tempo real. Nettelbeck [12] especifica uma frequência mínima de 20 a $30 \mathrm{~Hz}$ na atualização de cenas para que um ser humano possa ter a sensação de movimento contínuo ao visualizá-las.

\subsection{Realidade aumentada no ensino da geometria descritiva}

É possível encontrar relatos do uso da realidade aumentada especificamente para o ensino da geometria descritiva. As linhas de pesquisa mais comuns encontradas seguem:

- a criação de situações geométricas tridimensionais específicas, em ambientes animados;

- o desenvolvimento de ambiente colaborativo, normalmente empregando HMD;

- a avaliação da eficácia na aprendizagem e da aceitação dos alunos.

No Instituto de Computação Gráfica da Universidade de Tecnologia de Viena (Áustria) os ambientes colaborativos em realidade aumentada envolvendo esse fim foram relatados por Kaufmann et al. [13]. Nesse instituto foi desenvolvido o sistema pioneiro para ensino utilizando a realidade aumentada denominado Studierstube [14]. Questões técnicas de oclusão em ambientes colaborativos de realidade aumentada já eram abordadas no final da década de 1990 [15] nesse instituto, bem como nos Estados Unidos, no Laboratório de Tecnologias de Interfaces Humanas [16].

Em Kaufmann et al. [13] é descrita uma avaliação sobre o uso de sistemas envolvendo realidade em ambiente colaborativo para ministrar aulas de geometria descritiva. Para a avaliação foi utilizada a Isonorm, um conjunto normalizado de questões para a medição subjetiva da usabilidade de um sistema de software baseado na ISO 9241. Os autores obtiveram bons resultados, mas identificaram necessidades de melhorias no equipamento para que os alunos pudessem utilizar confortavelmente os HMD por vários minutos. Os autores colocam a realidade aumentada como uma ferramenta adicional para o ensino da geometria descritiva, sem dispensar os métodos convencionais analógicos ou, mesmo, a realidade virtual. Em Kaufmann e Csisinko [17] é relatado o desenvolvimento de um ambiente colaborativo onde 12 ou mais HMD podem ser utilizados simultaneamente, ligados a um computador.

Atualmente, no Brasil, Lima, Cunha e Haguenauer[18] apresentam um trabalho desenvolvido em realidade aumentada para a geração de vistas ortogonais de secções cônicas. No contexto do ensino a distância, os autores sugerem o uso da realidade aumentada para a geração de ambientes colaborativos. Oliveira et al. [19] afirmam que a colaboração é um dos aspectos mais importantes na aprendizagem.

O conceito de épura em realidade aumentada é citado por Seabra e Santos [20], porém, a representação da épura no sistema desenvolvido pelos autores não é feita da forma como mostrado nos livros de Príncipe Júnior [4], Souza Júnior [2] ou Ricca [5], mas, sim, como vistas ortogonais e aparentemente sem interação entre o objeto e a épura por movimentação de diferentes alvos no espaço. Em Seabra e Santos [20] foi feito um relato sobre um protótipo a ser desenvolvido para auxiliar no ensino da geometria, onde são mostrados os planos $\pi 1$ e $\pi 2$ da épura, mas somente em ambiente de realidade virtual.

Sem estarem voltados diretamente para o ensino da geometria descritiva, porém também voltados de forma geral ao ensino, podem ser encontrados vários relatos no Brasil, como, por exemplo, em [21], [22], [23], [24], [25], [26], entre outros. Internacionalmente, esse assunto é muito explorado.

\section{Elaboração do sistema}

O sistema desenvolvido neste trabalho pode ser classificado como sistema de vídeo para uso indoor. As cenas de realidade aumentada são mostradas no monitor do computador. O referenciamento em 3D é feito pela detecção 
automática de alvos nas imagens tomadas por uma webcam e pela posterior determinação das suas posições e orientações no espaço. Todo o procedimento é repetido de forma rápida o suficiente para dar a impressão de que ocorre em tempo real.

\subsection{Hardware e software}

Os seguintes itens de hardware foram utilizados:

- um microcomputador PC, com teclado, mouse, monitor e placa gráfica (Nvidia Geforce 7300 GS);

- uma câmara de vídeo Logitech Quickcam Communicate STX (VGA);

- uma impressora capaz de imprimir em formatos A4 e A3;

- suportes de madeira projetados especialmente para a aplicação, possíveis de serem visualizados nas figuras 2,3 e 4, nos quais são fixados planamente alvos para o software utilizado. A haste para a épura foi feita de forma a ficar em pé e as hastes para os objetos foram feitas de forma que se encaixem e possam deslizar entre si longitudinalmente, mantendo os alvos alinhados e variando a distância entre eles.

A lista do hardware necessário não é extensa. De fato, de posse dos alvos impressos, o desenvolvimento e o uso do programa requer apenas um computador (PC ou laptop) e uma webcam, equipamento que é barato e portátil. A qualidade de vídeo (resolução geométrica, radiométrica e de taxa de atualização), no entanto, deve ser adequada às condições de utilização do sistema.

A câmara deve passar por um processo de calibração, no qual são determinados os seus parâmetros de orientação interior e as distorções das lentes. Técnicas de calibração de câmaras são amplamente estudadas na fotogrametria. A calibração é feita por meio da medição de pontos homólogos em diferentes imagens tomadas de um mesmo objeto de dimensões conhecidas. Em Kato, Billinghurst e Poupyrev [27] ou na internet, em http://www.hitl.washington.edu/ artoolkit/documentation/usercalibration.htm, encontra-se descrito o procedimento de calibração de câmara utilizado no presente trabalho.

A lista de softwares utilizada é a seguinte:

- sistema operacional Linux OpenSuSE 11.1 (http://www.opensuse.org, licença GNU GPL), kernel 2.6.27.23-0.1default x86_64;

- biblioteca para desenvolvimento em realidade aumentada ARToolKit 2.71.3 (licença GNU GPL);

- biblioteca gráfica OpenGL 2.0 (licença SGI Free Software License B, versão 2.0); ${ }^{2}$

- IDE KDevelop para edição e compilação do programa (licença GNU LGPL);

- driver para a câmara de vídeo Generic Softwares Package for Camera Adapters (http://mxhaard.free.fr);

- interface GStreamer para receber a corrente de bytes da câmara de vídeo (http://gstreamer.freedesktop.org).

A base de desenvolvimento para o sistema proposto é a biblioteca ARToolKit (http://www.hitl.washington.edu/ artoolkit/), cujo uso exige a edição de códigos na linguagem de programação C. A renderização no ARToolKit é feita com o uso da biblioteca gráfica OpenGL.

\subsection{Visão geral das funcionalidades implementadas}

Pela idéia de representar a projeção em épura com realidade aumentada, o sistema deve ser capaz de ter a épura associada a um alvo e o objeto a ser projetado na épura em outro alvo. Cada alvo possui um sistema de coordenadas (ver seção 3.5) e deve ser possível fazê-los interagir.

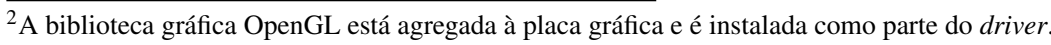


O código do programa exemplo do ARToolKit, relationTest, foi escolhido para servir de ponto de partida para o desenvolvimento neste trabalho. São aproveitados do programa as estruturas relacionadas à criação e ao controle das rotinas básicas de um programa de realidade aumentada, que são a tomada da imagem, o reconhecimento dos alvos, o cálculo da sua posição e orientação, a sua identificação pela figura interna, a aplicação das funções básicas de criação do ambiente em OpenGL com os devidos sistemas de coordenadas associados.

As funcionalidades necessárias são as seguintes:

1. definição dos alvos para a épura e para os objetos;

2. criação dos ambientes de projeção $3 \mathrm{D}$ e $2 \mathrm{D}$ com as respectivas janelas de visualização:

(a) no ambiente de realidade aumentada

i. criação da épura em um alvo,

ii. criação de objetos em dois outros alvos,

iii. transformação das coordenadas dos objetos para o sistema de coordenadas da épura,

iv. desenho das linhas de cota e de afastamento e dos arcos que descrevem a projeção dos pontos em 3D,

v. definição das variáveis de iluminação no ambiente virtual (OpenGL),

vi. desenho de todos os elementos virtuais utilizando as primitivas geométricas da OpenGL e o controle do ambiente virtual,

vii. renderização da cena com a épura e planos bissetores em transparência, sem poder ocluir nenhum outro objeto (OpenGL),

viii. suporte para rotação e translação dos objetos nos alvos, de forma a permitir a reconstituição de algumas situações geométricas especiais,

(b) no ambiente bidimensional da projeção de Monge:

i. obtenção das informações sobre os pontos projetados na épura em 3D,

ii. transformação das coordenadas 3D para a épura rebatida em 2D,

3. posicionamento dos textos explicativos sobre os elementos geométricos nas cenas de realidade aumentada e na épura rebatida em 2D.

\subsection{Preparação das janelas de visualização}

Para receber as cenas de realidade aumentada em três dimensões e para a representação em épura rebatida em duas dimensões, são criadas duas janelas de visualização separadas. A janela de visualização tridimensional é criada em todas as aplicações do ARToolKit, o que é normalmente feito ao ser invocada a função init () no método main. A janela adicional para a representação em $2 \mathrm{D}$ deve ser criada de forma explícita com a função glutcreateWindow.

Existindo duas janelas que devem ser renderizadas continuamente, devem ser especificadas de forma explícita as funções de renderização e resposta aos comandos de teclado relativas a cada uma delas. A renderização para uma ou outra janela é controlada pelo uso da função de OpenGL glutSetWindow, sendo dada a referência à janela específica como argumento (número inteiro).

O efeito do duplo buffer é necessário para a renderização contínua nas janelas de visualização e é ativado através do argumento GLUT_DOUBLE dada à função glutInitDisplayMode. É necessário invocar a função glut SwapBuffers após a conclusão das instruções de desenho nas janelas.

\subsection{Criação da épura e de alguns objetos virtuais}

A épura é desenhada no ambiente virtual a partir da definição dos pontos que formam os planos no espaço tridimensional. Isso é feito em OpenGL com o auxílio da primitiva gráfica GL_QUADS. A épura resultante é mostrada na Figura 2(a), sendo renderizada com efeito de transparência. Da mesma forma, pode ser feita a definição dos planos bissetores, que aparecem na mesma figura. Números romanos de I a IV mostram os números dos diedros. 
Os objetos a serem projetados são o ponto, a reta e o plano. O ponto é definido por uma coordenada no espaço $P(x, y, z)$. A reta, como representada em Príncipe Júnior [4], é definida por dois pontos $P_{1}\left(x_{1}, y_{1}, z_{1}\right)$ e $P_{2}\left(x_{2}, y_{2}, z_{2}\right)$. O plano, também como representado em Príncipe Júnior [4], é definido através das linhas de intersecção com os planos $\pi 1$ e $\pi 2$.

A épura é renderizada no sistema de coordenadas do alvo 0, ao passo que os objetos são renderizados nos alvos 1 e 2. A Figura 2(b) mostra os alvos dos dois objetos associados respectivamente a um ponto e a uma reta.

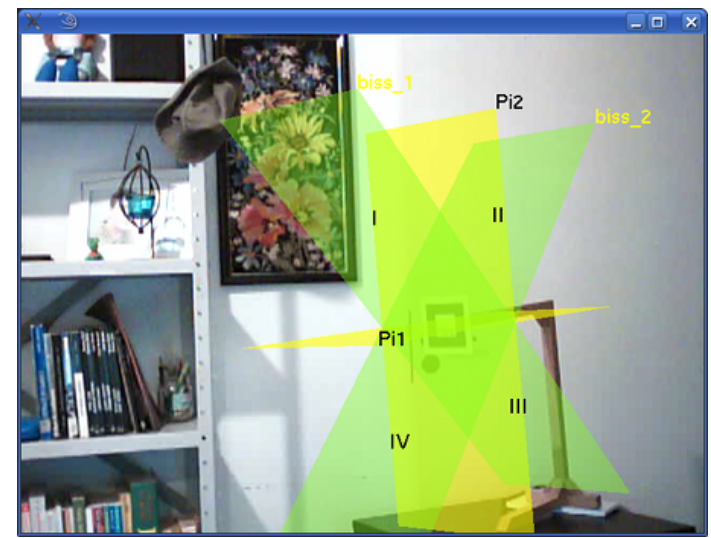

(a) Planos bissetores na cor verde adicionados ao ambiente, também com efeito de transparência

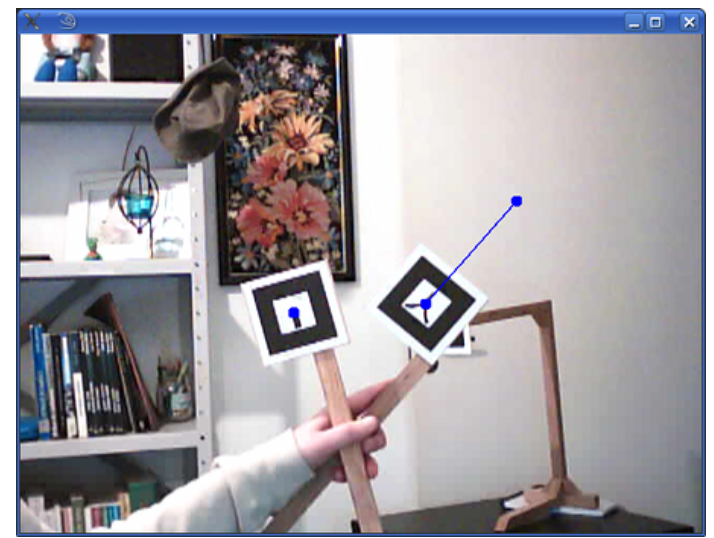

(b) Ponto e reta como objetos associados aos alvos

Figura 2: Representação dos objetos virtuais na cena

Na representação do ponto, este é definido na origem do sistema de coordenadas no alvo do objeto, sendo descrito na forma da Equação 1:

$$
P=(0,0,0)
$$

Na representação da linha, esta é formada pelo ponto $P 1$ na origem do sistema de coordenadas do alvo do objeto e por um ponto adicional P2 posicionado em um outro local, como mostram os modelos da Equação 2 . A variação da posição do ponto 2 pode ser controlada pelo usuário. Da mesma forma que para o ponto, as coordenadas que formam a linha são constantemente transformadas para o sistema de coordenadas da épura.

$$
P 1=(0,0,0) \quad P 2=(0,100,0)
$$

A modelagem do plano é mais complexa. Esse é modelado de forma infinita pela equação geral do plano, apresentada na Equação 3 [28]:

$$
a x+b y+c z+d=0
$$

Os valores de $a, b, c$ e $d$ podem ser calculados a partir de um ponto contido no plano e do vetor normal ao plano deste ponto. Entre os infinitos pontos e respectivos vetores que podem definir os planos $\pi 1$ e $\pi 2$ da épura são dadas as definiçõos das Equações 4 e 5:

$$
\begin{array}{ll}
P_{\pi 1}=(0,0,0) & \vec{N}_{\pi 1}=(0,0,1) \\
P_{\pi 2}=(0,0,0) & \vec{N}_{\pi 2}=(0,1,0)
\end{array}
$$

Esses planos passam pela origem do sistema de coordenadas da épura e possuem normais, respectivamente, na direção positiva de $z$ e na direção positiva de $y$. Suas equações se reduzem, respectivamente, para $z=0$ e $y=0$. 
O plano objeto pode ser definido pelos modelos da Equação 6, com a normal na direção positiva de $z$ sobre o alvo do objeto:

$$
P_{o b j}=(0,0,0) \quad \vec{N}_{o b j}=(0,0,1)
$$
7 e 8 :

Este plano é transformado para o sistema de coordenadas da épura, tendo sua descrição ali dada pelas Equações

$$
\begin{gathered}
P_{o b j_{T}}=\left(x_{P_{o b j_{T}}}, y_{P_{o b j_{T}}}, z_{P_{o b j_{T}}}\right) \\
\vec{N}_{o b j_{T}}=\left(x_{N_{o b j_{T}}}, y_{N_{o b j_{T}}}, z_{N_{o b j} T}\right)
\end{gathered}
$$

O valor de $d_{o b j_{T}}$ da equação geral do plano, é definido, assim, pela Equação 9:

$$
d_{o b j_{T}}=-x_{P_{o b j} T} \cdot x_{N_{o b j} T}-y_{P_{o b j} T} \cdot y_{N_{o b j} T}-z_{P_{o b j} T} \cdot z_{N_{o b j} T}
$$

As retas de intersecção do plano objeto transformado com os planos $\pi 1$ e $\pi 2$ podem ser assim reduzidas, respectivamente, para Equações 10 e 11:

$$
\begin{aligned}
& y_{\pi 1}=\frac{x_{\pi 1} \cdot x_{N_{o b j_{T}}}-d_{o b j_{T}}}{y_{N_{o b j} T}} \\
& z_{\pi 2}=\frac{x_{\pi 2} \cdot x_{N_{o b j_{T}}}-d_{o b j_{T}}}{z_{N_{o b j} j_{T}}}
\end{aligned}
$$

Para essas equações são dados os valores de $x_{\pi 1}$ e $x_{\pi 2}$, que formam as bordas da épura visualizada e são calculados os valores de $y_{\pi 1}$ e $y_{\pi 2}$.

Adicionalmente, são também renderizados os arcos de projeção dos pontos e linhas na épura. A renderização de arcos em OpenGL não é direta, porém, podem ser utilizadas funções trigonométricas que controlam a utilização repetida do desenho de linhas.

\subsection{Sistemas de coordenadas e transformações}

A épura se encontra representada junto ao alvo 0 e os objetos, junto aos alvos $1 \mathrm{e} 2$. Cada alvo possui um sistema de coordenadas, com origem no centro do alvo, com valores positivos de $x$ para a direita, com valores positivos de $y$ para cima e com valores positivos de $z$ para fora do plano do alvo. Os planos da épura são desenhados no sistema de coordenadas do alvo 0 e os objetos nos sistemas de coordenadas dos alvos 1 e 2.

Para que seja possível projetar os objetos na épura, é necessário que haja uma interação entre os sistemas de coordenadas. As coordenadas nos sistemas dos objetos devem ser transformadas para o sistema da épura. Essa transformação se dá por meio do sistema de coordenadas da câmara.

No funcionamento do ARToolKit estão envolvidas transformações de coordenadas entre espaços cartesianos e transformações em perspectiva. Estas últimas são utilizadas para relacionar o sistema de coordenadas da imagem com o da câmara de vídeo, durante a determinação da posição e orientação da câmara no espaço. Essa tarefa é realizada por rotinas prontas no ARToolKit que não foram estudadas neste trabalho. As transformações que interferem diretamente na elaboração do programa proposto são as que ocorrem entre os espaços cartesianos da épura e dos objetos.

Nas rotinas do ARToolKit é sempre utilizada a função arGet TransMat para relacionar os sistemas de coordenadas da câmara e dos objetos. A função retorna à posição e á orientação do alvo no sistema de coordenadas da câmara. Essa função é necessária para que os objetos possam ser representados no sistema de coordenadas da câmara ${ }^{3}$ com as funções do OpenGL.

\footnotetext{
${ }^{3}$ Mais informações sobre os sistemas de coordenadas e transformações no ARToolKit podem ser encontradas on-line nos links http://www.hitl. washington.edu/artoolkit/documentation/cs.htm e http://www.hitl.washington.edu/artoolkit/documentation/tutorialcamera.htm.
} 
No ARToolKit a matriz de transformação do objeto para a câmara $M_{O C}$ (objeto $\rightarrow$ câmara) retornada pela função arGet TransMat possui dimensão $3 \times 4$ (3 linhas e 4 colunas). Essas matrizes são transformadas para as coordenadas homogêneas do OpenGL no ARToolKit com o uso da função argConvGlpara. Pode ser observado que elementos das três primeiras colunas da matriz $M_{O C}$ são os elementos da matriz cartesiana de rotação $R$ que deve ser aplicada aos objetos dos alvos e que a quarta coluna (última) traz os elementos do vetor cartesiano de translação $T$, como mostra a Equação 12:

$$
M_{O C}=\left[\begin{array}{llll}
r_{11} & r_{12} & r_{13} & t_{14} \\
r_{21} & r_{22} & r_{23} & t_{24} \\
r_{31} & r_{32} & r_{33} & t_{34}
\end{array}\right]
$$

A transformação de coordenadas foi implementada utilizando coordenadas cartesianas, sendo primeiro aplicada a rotação e depois a translação. Ambos os espaços nessa transformação, de origem e de fim, são cartesianos. A transformação de um ponto no alvo do objeto para o sistema de coordenadas da épura deve passar pelo sistema de coordenadas da câmara e é composta pela aplicação consecutiva de duas transformações:

1. do objeto para a câmara, onde a matriz de transformação $M_{O C}$ é dada pelo uso da função arGetTransMat;

2. da câmara para a épura, onde a matriz $M_{E C}$ (épura $\rightarrow$ câmara) é obtida pelo uso da função arGet TransMat e depois é invertida pelo uso da função arUtilMat Inv do ARToolKit, gerando a matriz $M_{C E}$ (câmara $\rightarrow$ épura).

Assim, a matriz de transformação do objeto para a épura $M_{O E}$ (objeto $\rightarrow$ épura) é dada pela Equação 13:

$$
M_{O E}=M_{O C} \cdot M_{C E}
$$

A matriz retornada pelo uso da função arUti lMat Inv possui também dimensão $3 \times 4$ e traz a mesma estrutura da matriz de transformação dada na Equação 12. Para efetuar a multiplicação de matrizes, consta no ARToolKit a função arUtilMatMul, que é utilizada para gerar a matriz $M_{O E}$ também na mesma estrutura da Equação 12.

Com isso, a matriz de rotação do objeto para a épura $R$ é composta pelos elementos das três primeiras colunas da matriz $M_{O E}$ e o vetor de translação $T$ é obtido pelos elementos da quarta coluna. A transformação de um ponto $P_{O}\left(x_{O}, y_{O}, z_{O}\right)$ no alvo de um dos objetos (1 ou 2) para o ponto $P_{E}\left(x_{E}, y_{E}, z_{E}\right)$ no sistema de coordenadas da épura no alvo 0 é dada então pela Equação 14:

$$
P_{E}=P_{O} \cdot R+T
$$

ou, em forma matricial (Equação 15):

$$
\left[\begin{array}{l}
x_{E} \\
y_{E} \\
z_{E}
\end{array}\right]=\left[\begin{array}{lll}
r_{11} & r_{12} & r_{13} \\
r_{21} & r_{22} & r_{23} \\
r_{31} & r_{32} & r_{33}
\end{array}\right] \cdot\left[\begin{array}{l}
x_{O} \\
y_{O} \\
z_{O}
\end{array}\right]+\left[\begin{array}{c}
t_{1} \\
t_{2} \\
t_{3}
\end{array}\right]
$$

De posse das coordenadas transformadas, a projeção para a épura rebatida é feita simplesmente utilizando os valores de $x$ e $-z$ para o plano $\pi 1$ e de $x$ e $y$ para o plano $\pi 2$, na janela de visualização bidimensional.

O efeito resultante da transformação das coordenadas do objeto para o sistema de coordenadas da épura é a interação entre eles, onde os movimentos aplicados ao objeto pela movimentação do alvo são representados no sistema de coordenadas da épura pelo desenho das linhas de cota e de afastamento. Simultaneamente são calculadas as posições dos pontos bidimensionais na épura rebatida, que acompanha a interação. Nas figuras da Seção 4 são dados vários exemplos onde essa interação é demostrada. 


\section{Uso do sistema}

As cenas mostradas em um SRA são continuamente atualizadas, dando a impressão de que o computador acompanha em tempo real as alterações das posições e orientações dos alvos no espaço. Tal dinamicidade não é possível de ser representada no papel. Por isso, ao analisar os exemplos de uso do SRA trazidos nesta seção, o leitor deve ter em mente que as figuras que vê não são estáticas. A demonstração da propriedade da atualização em tempo real deve ser demonstrada com o sistema em funcionamento.

Para ser utilizado o sistema, o alvo da épura deve ser posicionado na cena de forma que seja reconhecido nas imagens tomadas pela câmara. Pode ser deixado em algum local fixo em que os alvos dos objetos estejam se movimentando ao seu entorno. Uma vez que o alvo da épura e o alvo de algum objeto são simultaneamente detectados, as respectivas linhas de projeção são mostradas na cena. Simultaneamente, tudo é mostrado na épura rebatida para duas dimensões. Durante a movimentação dos alvos, estes devem estar sempre visíveis à câmara, caso contrário, a projeção é interrompida até que sejam novamente visualizados. A detecção dos alvos e o consequente referenciamento em 3D ocorre de forma estável.

Foi verificado que as condições de iluminação interferem na capacidade de o sistema reconhecer os alvos na cena. Esse efeito pode variar de acordo com as características da câmara e com o posicionamento e a intensidade da luz ambiente. Os alvos foram facilmente reconhecidos em ambientes de iluminação moderada: um ambiente sem penetração de luz solar direta, com uma lâmpada incandescente de teto com 100W. Mesmo nessas condições, a variação do ângulo formado entre a normal ao plano do alvo e a direção dos raios luminosos também interfere, dificultando a identificação quando o ângulo se aproxima de zero (máxima exposição).

O sistema oferece a possibilidade da edição das propriedades de alguns elementos da cena, o que é feito via teclado, com uma relação de comandos disponíveis. Sua exploração possibilita a geração de cenas como as mostradas na Figura 3. Durante a operação do sistema convém ter uma das mãos livres para operar o teclado.

São demonstradas, na Figura 3, algumas situações especiais envolvendo os objetos. Na Figura 3(a) é mostrada a projeção de um ponto sobre uma linha, sendo os alvos ajustados adequadamente. Movimentando os alvos no espaço (fixos entre si) as projeções dos objetos se alteram em tempo real, mas mantendo sempre a situação de pertinência de ponto a reta: as projeções do ponto estão sobre as projeções de mesmo nome da reta (horizontal sobre horizontal, vertical sobre vertical). As cores atribuídas às projeções horizontal e vertical auxiliam nessa verificação (verde com verde, vermelho com vermelho).

Na Figura 3(b) são mostradas duas retas paralelas. Ao serem movimentadas no espaço, elas mantêm na épura sempre alguma condição de paralelismo, conforme traz Príncipe Júnior [3]:

1. as suas projeções de mesmo nome são paralelas (caso da figura);

2. duas projeções de mesmo nome se confundem e as outras duas são paralelas;

3. as suas projeções sobre um mesmo plano se reduzem, cada uma, a um ponto.

Na Figura 3(c) é representada uma reta vertical em um plano vertical, cuja épura obtida é similar à mostrada em Príncipe Júnior [3] para a situação específica.

A Figura 4 mostra o sistema sendo utilizado dentro de uma sala de aula. Um laptop é utilizado para executar o programa, cujas imagens são tomadas por uma câmara nele embutida. Este é posicionado em um lugar mais alto, de forma que possa captar o alvo da épura na imagem com uma certa inclinação em relação aos seus dois planos. Dessa forma, podem ser claramente visualizadas as linhas de projeção que ligam os objetos à épura. As hastes com os alvos para os objetos estão posicionadas de forma a criar um ponto sobre uma reta. As imagens geradas pelo sistema são projetadas em uma tela com o auxílio de um projetor conectado ao computador.

\section{Conclusões e perspectivas}

Neste trabalho foi apresentado um sistema capaz de representar a épura no espaço tridimensional, as primitivas ponto, reta e plano e os elementos representativos das projeções nos planos horizontal e vertical. Simultaneamente, o sistema representa a respectiva épura rebatida em duas dimensões. 


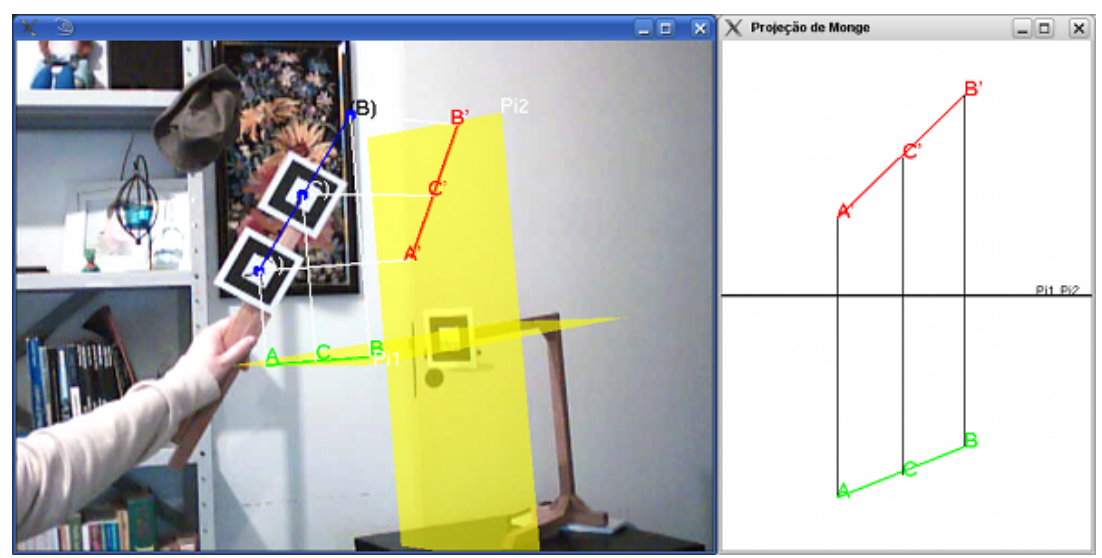

(a) Ponto sobre linha

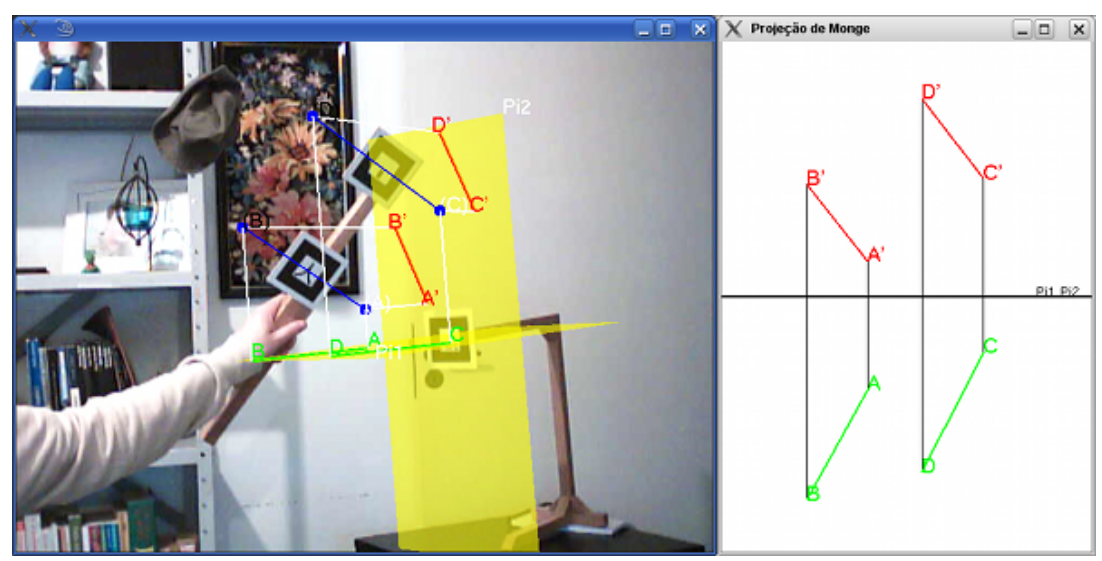

(b) Linhas paralelas

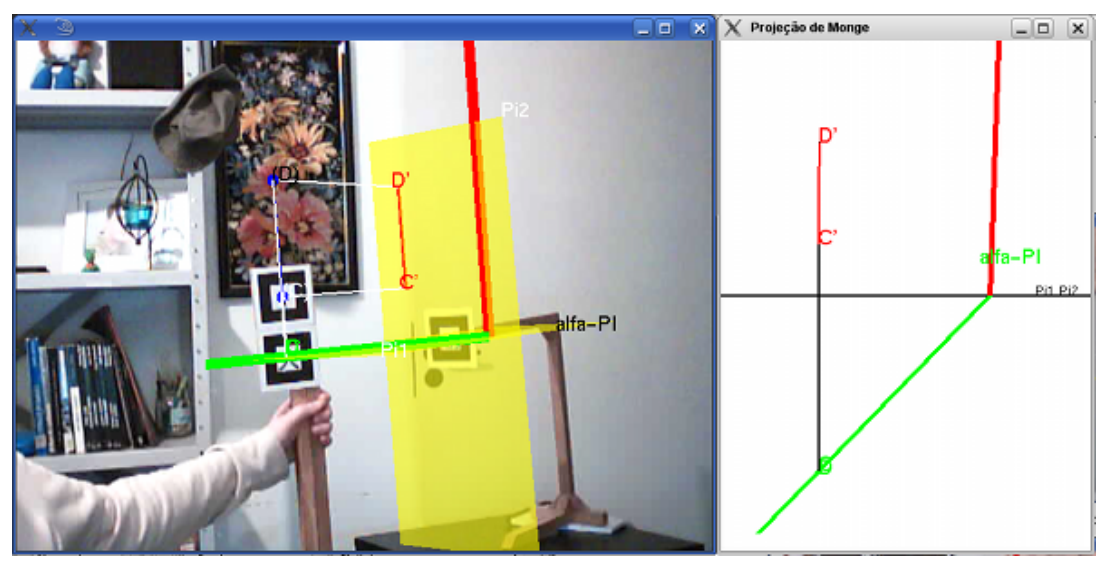

(c) Reta vertical em plano vertical

Figura 3: Demonstrações com combinações de ponto, reta e plano 


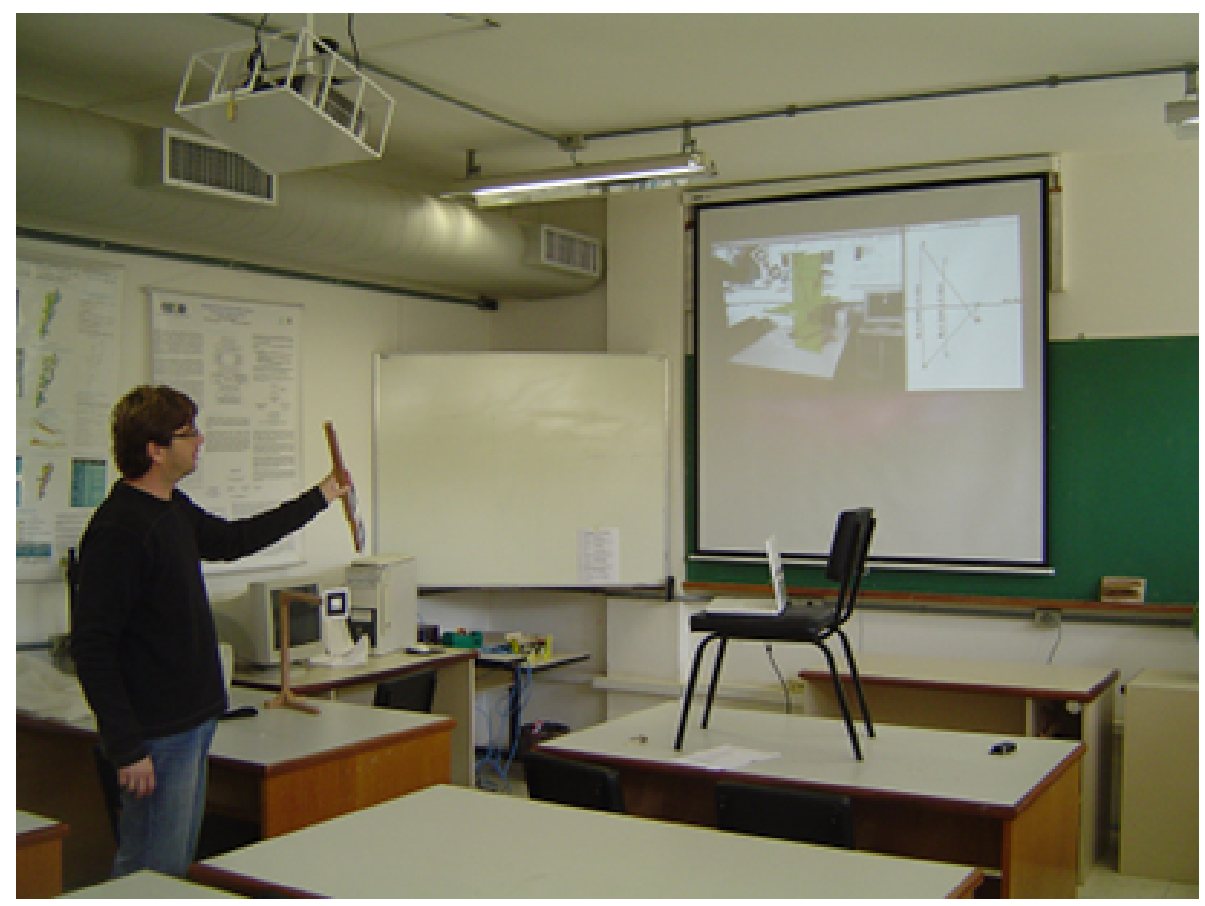

Figura 4: Demonstração em sala de aula

O SRA desenvolvido pode ser utilizado como uma ferramenta adicional para o entendimento da projeção de Monge no ensino da geometria descritiva. Um aumento na eficácia do ensino e a aceitação do uso do sistema por parte de alunos no ambiente de sala de aula devem ser futuramente avaliados. Porém, como mostram, por exemplo, os resultados obtidos por Kaufmann et al. [13], a ideia é promissora. Com o sistema desenvolvido neste trabalho, não há problemas de cansaço relacionados pelo uso do HMD, como relatado em Kaufmann et al. [13]. Porém, o manuseio das hastes para posicionar os alvos dos objetos no entorno da épura virtual causa cansaço, particularmente para manter as situações geométricas especiais de relacionamento entre os objetos, para que possam ser analisadas pelos usuários. Para minimizar esse cansaço, pode ser incluído no sistema um comando de congelamento da cena, para ser acionado no momento em que a situação desejada for atingida.

Como característica do sistema desenvolvido, o posicionamento dos alvos no espaço foi feito pela movimentação das hastes, o que pode tornar mais intuitivo o entendimento da cena gerada. A configuração dos alvos na cena, porém, sofre limitações de posicionamento. Os alvos devem estar sempre posicionados de forma que possam ser reconhecidos nas imagens da câmara de vídeo, o que pode dificultar a configuração de determinadas cenas. Em um ambiente de realidade virtual essa não seria uma limitação.

Este trabalho também mostrou que um SRA pode ser desenvolvido sendo utilizados exclusivamente softwares livres. Essa característica é particularmente interessante em instituições de pesquisa, sendo demonstrado aos interessados que há ferramentas gratuitas que podem auxiliar no desenvolvimento de ideias relacionadas com o tema.

Para o desenvolvimento futuro do sistema, sugere-se:

- uso de funcionalidades de animação da OpenGL para reprodução automática do desenvolvimento de operações em épura (por exemplo, pertinência de ponto a plano) ao posicionar os objetos em determinadas posições e orientações e sendo congelada a imagem. Isso pode ser feito pela definição de uma função que gera as alterações de coordenadas, que são dadas para a renderização;

- implementação de funcionalidade para o carregamento de objetos a partir de arquivos em VRML. Com isso poderiam ser criados, por exemplo, modelos em CAD das hastes que suportam os alvos. A importação para o ambiente do sistema poderia se dar via OpenVRML. A inclusão de objetos a partir de arquivos em VRML é relatada, por exemplo, em Cipriano e Bila [29]; 
- implementação de funcionalidade para processamento de oclusão. Para isso poderiam ser utilizados os modelos virtuais das hastes. Nesse caso as suas geometrias poderiam ser comparadas com a da épura na cena virtual (por utilização de $z$-buffer) e, assim, as hastes poderiam ocluir a épura, aumentando o realismo da cena;

- implementação de objetos mais complexos, como poliedros, que poderiam ser renderizados na cena como sólidos ou em estrutura de arame (wireframe).

\section{Referências}

[1] Virgilio Athayde Pinheiro. Noções de geometria descritiva. Ao Livro Técnico, Rio de Janeiro, 2 edition, 1968.

[2] Hugo de Andrade de Souza Júnior. Geometria descritiva e perspectiva. Pioneira, São Paulo, 1975.

[3] Alfredo dos Reis Príncipe Júnior. Noções de geometria descritiva, volume 2. Nobel, São Paulo, 25 edition, 1980.

[4] Alfredo dos Reis Príncipe Júnior. Noções de geometria descritiva, volume 1. Nobel, São Paulo, 34 edition, 1983. ISBN 85-213-0163-4.

[5] Guilherme Ricca. Geometria descritiva - Método de Monge. Fundação Calouste Gulbenkian, Lisboa, 2 edition, 2000. ISBN: 972-31-0547-0.

[6] Ronald Azuma. A survey of augmented reality. In Presence: Teleoperators and Virtual Environments, volume 6, pages 355 - 385, August 1997.

[7] Cláudio Kirner and Tereza G. Kirner. Virtual Reality and Augmented Reality Applied to Simulation Visualization, In: Simulation and Modeling: Current Technologies and Applications, volume 1. Hershey-NY: IGI Publishing, 1 edition, March 2008.

[8] Ronald Azuma, Y. Baillot, R. Behringer, S. Feiner, S. Julier, and B. MacIntyre. Recent advances in augmented reality. In IEEE Computer Graphics and Applications, volume 25 (6), pages 24-35, Nov-Dec 2001.

[9] Alexandre Hering Coelho. Erweiterte Realität zur Visualisierung simulierter Hochwasserereignisse. PhD thesis, Universität Karlsruhe (TH), Fakultät für Bauingenieur-, Geo- und Umweltwissenschaften, Karlsruhe, Deutschland, Dezember 2004.

[10] Alexandre Hering Coelho and Hans-Peter Bähr. Visualização de dados de CAD e LIDAR por meio de Realidade Aumentada. In Anais XII Simpósio Brasileiro de Sensoriamento Remoto, INPE, pages 2925-2932, Goiânia, Brasil, abril 2005.

[11] Ivan E. Sutherland. A head-mouted three-dimensional display. In AFIPS Conference, volume 33, pages 757 764, 1968.

[12] Bernd Nettelbeck. Zeitlich gesteuerte änderung von darstellungsstilen. Diplom thesis at the department of simulation and graphics, Otto-von-Guericke-Universität, Magdeburg, Germany, 2003.

[13] Hannes Kaufmann, Karin Steinbügl, Andreas Dünser, and Judith Glück. Improving spatial abilities by geometry education in augmented reality - application and evaluation design. In VRIC Laval Virtual 2005 Proceedings, pages 25-34. IEEE, 2005.

[14] Dieter Schmalstieg, Anton L. Fuhrmann, Gerd Hesina, Zsolt Szalavári, L. Miguel Encarnação, Michael Gervautz, and Werner Purgathofer. The studierstube augmented reality project. Presence, 11(1):33-54, 2002.

[15] Anton Fuhrmann, Gerd Hesian, François Faure, and Michael Gervautz. Occlusion in collaborative augmented environments. Computers and graphics, 23(6):809-819, december 1999. http://www.cg.tuwien.ac.at/research/ vr/occlusion.

[16] M. Billinghurst, S. Weghorst, and T. A. III Furness. Shared Space: An Augmented Reality Approach for Computer Supported Collaborative Work, pages 25-36. Number 3. Springer Verlag, 1998.

[17] H. Kaufmann and M. Csisinko. Multiple head mounted displays in virtual and augmented reality applications. The International Journal of Virtual Reality, 2(6):43-50, 2007. 
[18] Álvaro José Rodrigues de Lima, Gerson Gomes Cunha, and Cristina Jasbinschek Haguenauer. Realidade aumentada no ensino de geometria descritiva. Realidade Virtual, (1):2, agosto 2008. ISSN: 1984-0179.

[19] Francisco César de Oliveira, Claudio Kirner, Cleberson Eugenio Forte, and Rafael Santin. Colaboração em ambientes educacionais com realidade aumentada. RENOTE - Revista Novas Tecnologias na Educação, 7(1), Julho 2009. No prelo.

[20] R. D. Seabra and E. T. Santos. Utilização de técnicas de realidade virtual no projeto de uma ferramenta 3d para desenvolvimento da habilidade de visualização espacial. Educação Gráfica, 9(9):111-122, 2005. Bauru.

[21] L. A. Consularo, N. Calonego Jr, C. A. Dainese, T. R. Garbin, C. Kirner, J. Trindade, and C. Fiolhais. ARToolKit: Aspectos Técnicos e Aplicações Educacionais, pages 141-183. SBC, São Paulo, 2004.

[22] E. R. Zorzal, Alexandre Cardoso, Claudio Kirner, and Edgard Lamounier Júnior. Realidade aumentada aplicada em jogos educacionais. In V Workshop de Educação em Computação e Informática do Estado de Minas Gerais - WEIMIG, Ouro Preto, 2006.

[23] Claudio Kirner and E. R. Zorzal. Aplicações educacionais em ambiente colaborativos com realidade aumentada. In SBIE2005 - XVI Simpósio Brasileiro de Informática na Educação, volume 1, Juíz de Fora-MG, 2005.

[24] A. J. R. Lima, G. G. Cunha, C. Haguenauer, and L. G. R. Lima. Torus surfaces of descriptive geometry in augmented reality. In 5 Workshop de Realidade Virtual e Aumentada, volume 1, pages 1-4, Bauru, 2008.

[25] Roger Luz, Marcos Wagner Souza Ribeiro, Alexandre Cardoso, Edgard Lamounier Jr., Hugo Rocha, and Wender Silva. Análise de aplicações de realidade aumentada na educação profissional: Um estudo de caso no senai DR/GO. In $5^{\circ}$ Workshop de Realidade Virtual e Aumentada, Bauru, 2008.

[26] A. Cardoso, Edgard A Lamounier Júnior, and Márcio Antônio Duarte. O uso de realidade aumentada no ensino de física. In II Workshop sobre Realidade Aumentada, volume 1, pages 01-04, Piracicaba, 2005.

[27] Hirokazu Kato, Mark Billinghurst, and Ivan Poupyrev. ARToolKit, version 2.33, 2000.

[28] Alfredo Steinbruch and Delmar Basso. Geometria analitica. McGraw-Hill, São Paulo, 1991. ISBN 0074504096.

[29] Bruno Cipriano and Vasco Bila. Associar um modelo 3D a um marker usando o exemplo simpleVRML, 2007. MovLab - Universidade Lusófona de Humanidades e Tecnologias, Lisboa, Portugal. 